QUARTERLY OF APPLIED MATHEMATICS

VOLUME LXIX, NUMBER 1

MARCH 2011, PAGES 15-38

S 0033-569X(2010)01198-3

Article electronically published on December 9, 2010

\title{
ENERGETIC PROPERTIES OF THE ELASTIC HALF-SPACE LOADED BY A PERIODIC DISTRIBUTION OF VIBRATING PUNCHES: THE STUDY OF THE PHASE SHIFT INFLUENCE
}

\author{
BY \\ EDOARDO SCARPETTA (D.I.I.M.A., University of Salerno, 84084 Fisciano (SA), Italy) \\ AND \\ MEZHLUM A. SUMBATYAN (Faculty of Mechanics and Mathematics, Rostov State University, \\ 344090 Rostov-on-Don, Russia)
}

\begin{abstract}
We develop an analytical approach to study the wave properties of an elastic half-space subjected to harmonic vibrations applied on its free surface by a periodic array of rigid punches. Contrary to previous investigations, it is assumed that any neighbouring pair of these oscillates with a (common) phase shift, thus implying rather specific behaviours of the structure. Starting from integral equations for the contact stress and representation formulas for the wave field in both the anti-plane and in-plane problems, suitable mild approximations on the kernels allow analytical solutions of some related (auxiliary) integral equations in a given range of (not too high) frequency.

The explicit formulas thus obtained for the wave field are reflected through some figures and enable us to investigate the energetic properties of the structure with respect to different phase shifts. A direct numerical solution of the original integral equations confirms the precision of the analytical solution.
\end{abstract}

1. Introduction. As is well known, the transfer of force or energy from one elastic body to another is a problem of great relevance in both mechanical and civil engineering, where the theory and the practice of Classical Elasticity are deeply involved. Nowadays, one of the most important applications is probably connected with certain problems arising in Applied Geophysics, more precisely in the study of seismic vibrations for protection of the building foundations, and in the ground exploration activities for geological (mining) research [1, 2].

As an example of the latter topic (in addition to other methods), the researchers used to apply a vibrator on the ground surface to generate a certain structure of waves inside the earth, in order to evaluate the mechanical properties of soils and/or discover expected deposits of coal, oil or natural gases. Initially, one massive vibrator (so-called punch)

Received April 2, 2009.

2010 Mathematics Subject Classification. Primary 74J99.

(C)2010 Brown University 
was used for this purpose; however, some recent results (reflected in numerous patents; see, e.g., [3] - [5] ) seem to show that the efficiency of energy transmission into the soil can be improved by using several punches vibrating simultaneously. Actually, it comes out from the practice that multiple sources and receiver arrays provide a good technique for high-resolution results [6]; this can be explained by noting that in such a case one can arrange an optimal control of the frequency, amplitude or phase distribution along the punches' array, thus achieving the greatest efficiency from such systems in energy and resolution.

Crucial for these results can be a suitable phase shift between adjacent vibrators. In a previous paper [7, we studied the wave propagation through an elastic half-space originated by a periodic distribution of rigid (identical) punches vibrating above its free surface in the case of a null phase shift, namely, when all punches oscillate with the same phase (in addition to the same amplitude and frequency); in this paper, we aim to treat the case of a common phase shift between any pair of adjacent punches, which, as will be clear from the results obtained, implies very interesting energetic properties of the vibrating structure.

After recalling the formulation of both the anti-plane and in-plane problems in this context (Section 2), we work out the integral equations and representation formulas in which such problems are reflected for an arbitrary phase shift (Section 3). Then, by selecting suitable ranges of frequency according to the given phase shift, we will reduce the problems to some integral equations which are independent of frequency and arise from mild approximations of the original kernels valid in those ranges (Section 4). Such (auxiliary) integral equations are solved analytically (Section 5), so that an explicit representation, with respect to frequency, can be set up for the wave field in all the considered cases (Section 6). In the final Section 7, the peculiar properties of the structure will be pointed out and discussed, by also looking at some graphs in which the analytical results can be reflected for concrete values of the physical and geometric parameters. Parallel to this approach, a direct numerical method will be applied to the original (exact) integral equations in order to control the validity of the (approximate) analytical solution in the given frequency ranges.

2. Formulation of the anti-plane and in-plane problems. Reduction to integral equations. Let us reconsider the oscillating structure treated in [7]: we have an infinite, periodic distribution of rigid coplanar punches lying over the free surface of an elastic half-space $y \geq 0$. The punches can vibrate harmonically with given amplitude, frequency and (possibly different) phases, thus generating a wave propagation through the half-space; such punches are infinitely long (in the $z$-direction), $2 b$ is the common width of their bases and $2 a$ the period of the array $(a>b)$. If we denote by $S \times\{-\infty<z<+\infty\}$ the total contact area between the punches and the half-space surface, in the (geometrically) periodic problem we are treating it follows that

$$
S=\bigcup_{n=-\infty}^{+\infty}(-b+2 a n, b+2 a n) .
$$

Figure 1 shows the section of the structure with (any) normal plane $x y$. 


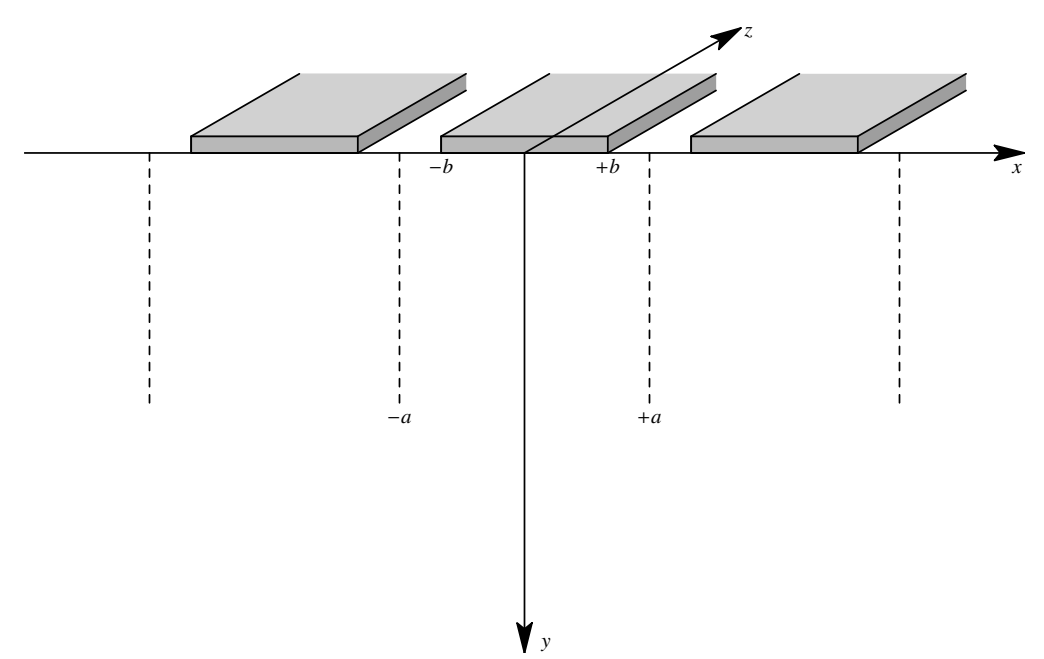

FIG. 1. A periodic array of rigid punches (of width $2 b$ ) vibrating above the free surface of an elastic half-space. The period is $2 a$.

In the anti-plane problem, we assume that each punch is perfectly joined with the half-space, and the applied forces are also directed along the $z$-axis. In the in-plane problem, we assume that the contact between the punch and the half-space is frictionless, and the applied forces are directed along the $y$-axis, namely, normally onto the punches. In both cases, this clearly implies a displacement of given amplitude at $y=0$ (in the contact zones), which will be involved as a boundary datum in our problems.

Of course, if $\omega$ is the (angular) frequency of the harmonic applied forces, the linear model here adopted implies that time dependence is harmonic with the same $\omega$ throughout the whole structure; thus, in the sequel the common factor $e^{-i \omega t}$ should be understood in all field variables.

By classical results of Elasticity Theory [7, 8, the anti-plane (scalar) problem in the present context can be reduced to an integral equation and a representation formula, as follows:

$$
\begin{gathered}
\int_{S} \tau(\xi)\left[\int_{-\infty}^{+\infty} \frac{e^{-i \alpha(x-\xi)}}{\sqrt{\alpha^{2}-k^{2}}} d \alpha\right] d \xi=-2 \pi \mu w_{0}, \quad x \in S \\
w(x, y)=-\frac{1}{2 \pi \mu} \int_{S} \tau(\xi)\left[\int_{-\infty}^{+\infty} \frac{e^{-\sqrt{\alpha^{2}-k^{2}} y}}{\sqrt{\alpha^{2}-k^{2}}} e^{-i \alpha(x-\xi)} d \alpha\right] d \xi \quad, \quad y \geq 0 .
\end{gathered}
$$

In such relations, we use the following notation:

$k$ is the transverse wave number, namely $k=\omega \sqrt{\rho / \mu}$, where $\rho$ and $\mu$ are the mass density and shear modulus of the elastic material in the half-space, respectively.

$w$ is the stationary wave field, namely $w(x, y)=u_{z}(x, y, t) e^{i \omega t}$, where $u_{z}$ is the outof-plane displacement component (only non-null and clearly independent of $z$ ); $w_{0}=$ $w(x, 0), x \in S$, denotes the common amplitude of the (horizontal) punches' vibration. 
$\tau$ is the tangential stress over the half-space surface, namely $\left.\tau(x) \equiv \mu \frac{\partial w(x, y)}{\partial y}\right|_{y=0}$, $x \in S$ (of course, $\tau=0$ outside $S$ ).

Along parallel lines, the in-plane (vector) problem can be reduced to the following integral equation and representation formulas:

$$
\begin{aligned}
& \int_{S} \sigma(\xi)\left[\int_{-\infty}^{+\infty} \frac{\sqrt{\alpha^{2}-k_{1}^{2}}}{\Delta(\alpha)} e^{-i \alpha(x-\xi)} d \alpha\right] d \xi=\frac{2 \pi \mu}{k_{2}^{2}} u_{0}, \quad x \in S \\
& u_{x}(x, y)=\frac{i}{2 \pi \mu} \int_{S} \sigma(\xi)\left\{\int _ { - \infty } ^ { + \infty } \frac { \alpha } { \Delta ( \alpha ) } \left[\left(k_{2}^{2}-2 \alpha^{2}\right) e^{-\sqrt{\alpha^{2}-k_{1}^{2}} y}\right.\right. \\
& \left.\left.+2 \sqrt{\alpha^{2}-k_{1}^{2}} \sqrt{\alpha^{2}-k_{2}^{2}} e^{-\sqrt{\alpha^{2}-k_{2}^{2}} y}\right] e^{-i \alpha(x-\xi)} d \alpha\right\} d \xi, \quad y \geq 0 \\
& u_{y}(x, y)=\frac{1}{2 \pi \mu} \int_{S} \sigma(\xi)\left\{\int _ { - \infty } ^ { + \infty } \frac { \sqrt { \alpha ^ { 2 } - k _ { 1 } ^ { 2 } } } { \Delta ( \alpha ) } \left[\left(k_{2}^{2}-2 \alpha^{2}\right) e^{-\sqrt{\alpha^{2}-k_{1}^{2}} y}\right.\right. \\
& \left.\left.+2 \alpha^{2} e^{-\sqrt{\alpha^{2}-k_{2}^{2}} y}\right] e^{-i \alpha(x-\xi)} d \alpha\right\} d \xi, \quad y \geq 0 .
\end{aligned}
$$

In such relations, we define:

$k_{1}$ and $k_{2}$ are the longitudinal and transverse wave numbers, namely $k_{1}=\omega \sqrt{\rho /(\lambda+2 \mu)}$, $k_{2}=\omega \sqrt{\rho / \mu}\left(>k_{1}\right)$, where $\lambda$ and $\mu$ are the Lamé moduli of the elastic material $(\rho, \mu$ as before).

$\Delta(\alpha)$ is a pertinent Rayleigh function, namely

$$
\Delta(\alpha)=\left(2 \alpha^{2}-k_{2}^{2}\right)^{2}-4 \alpha^{2} \sqrt{\alpha^{2}-k_{1}^{2}} \sqrt{\alpha^{2}-k_{2}^{2}} .
$$

$u_{x}$ and $u_{y}$ are the components of the stationary displacement field (only non-null and independent of $z$ ); $u_{0}=u_{y}(x, 0), x \in S$, denotes the common amplitude of the (vertical) punches' vibration.

$\sigma$ is the normal stress over the half-space surface, namely $\left.\sigma(x) \equiv \lambda \frac{\partial u_{x}(x, y)}{\partial x}\right|_{y=0}+$ $\left.(\lambda+2 \mu) \frac{\partial u_{y}(x, y)}{\partial y}\right|_{y=0}, x \in S$ (of course, $\sigma=0$ outside $S$ ).

Once having resolved the integral equations for $\tau$ or $\sigma$, the representation formulas can be used to give the structure of the wave field throughout the elastic half-space.

3. The case of a phase shift between adjacent punches in the periodic distribution. We now focus our attention on the integral equations stated in the previous section, which, as they stand, are clearly valid for an arbitrary contact area $S$. Let us take into account that $S$ is given as in (2.1), and assume that, by a suitable choice of the applied forces, there is a phase shift $\varepsilon \in[0,1)$ between the vibration of two adjacent punches: we mean by this that $\tau$ (or $\sigma$ ) in $\xi+2$ an is equal to $\exp (2 i \pi \varepsilon n) \times \tau$ (or $\sigma$ ) in $\xi$. Note that, if $\varepsilon$ is a rational number, say $\varepsilon=p / q$, the problem is fully periodic and the period remains 2aq; on the contrary, if $\varepsilon$ is irrational, the problem is not periodic (or better, is periodic only from the geometrical point of view). 
As a consequence, looking at the integrals over $S$ contained in (2.2)-(2.5), we have

$$
\begin{gathered}
\int_{S}\left(\begin{array}{c}
\tau(\xi) \\
\sigma(\xi)
\end{array}\right) e^{i \alpha \xi} d \xi=\sum_{n=-\infty}^{+\infty} \int_{-b+2 a n}^{b+2 a n}\left(\begin{array}{c}
\tau(\xi) \\
\sigma(\xi)
\end{array}\right) e^{i \alpha \xi} d \xi \\
=\sum_{n=-\infty}^{+\infty} e^{2 i \pi \varepsilon n} \int_{-b}^{b}\left(\begin{array}{c}
\tau(\xi) \\
\sigma(\xi)
\end{array}\right) e^{i \alpha(\xi+2 a n)} d \xi \\
=\frac{\pi}{a} \sum_{m=-\infty}^{+\infty} \delta[\alpha-\pi(m-\varepsilon) / a] \int_{-b}^{b}\left(\begin{array}{c}
\tau(\xi) \\
\sigma(\xi)
\end{array}\right) e^{i \alpha \xi} d \xi
\end{gathered}
$$

where the following formula (implied by well-known properties of the Dirac function $\delta$; see [9]) has been used:

$$
\sum_{n=-\infty}^{+\infty} e^{2 i n(\pi \varepsilon+a \alpha)}=\frac{\pi}{a} \sum_{m=-\infty}^{+\infty} \delta[\alpha-\pi(m-\varepsilon) / a] .
$$

Thus, the integral equation (2.2) and the representation formula (2.3) for the anti-plane problem can be rewritten as follows:

$$
\begin{gathered}
\int_{-b}^{b} K^{\tau}(x-\xi) \tau(\xi) d \xi=C^{\tau}, \quad x \in(-b, b) \\
w(x, y)=-\frac{1}{2 a \mu} \sum_{m=-\infty}^{+\infty} \frac{e^{-q_{m}^{\tau} y}}{q_{m}^{\tau}}\left(\int_{-b}^{b} \tau(\xi) e^{i \pi(m-\varepsilon) \xi / a} d \xi\right) e^{-i \pi(m-\varepsilon) x / a}, \quad y \geq 0
\end{gathered}
$$

where the kernel $K^{\tau}$ is given by

$$
K^{\tau}(x)=\sum_{m=-\infty}^{+\infty} \frac{1}{a q_{m}^{\tau}} e^{-i \pi(m-\varepsilon) x / a},
$$

and we put

$$
C^{\tau}=-2 \mu w_{0}, \quad q_{m}^{\tau}=\sqrt{[\pi(m-\varepsilon) / a]^{2}-k^{2}} .
$$

Similarly, (2.4) and (2.5) for the in-plane problem become

$$
\begin{aligned}
& \int_{-b}^{b} K^{\sigma}(x-\xi) \tau(\xi) d \xi=C^{\sigma}, \quad x \in(-b, b), \\
& u_{x}(x, y)=\frac{i}{2 a \mu} \sum_{m=-\infty}^{+\infty} \frac{\pi(m-\varepsilon)}{a \Delta_{m}}\left\{\left[k_{2}^{2}-2\left[\frac{\pi(m-\varepsilon)}{a}\right]^{2}\right] e^{-q_{m}^{\sigma} y}+2 q_{m}^{\sigma} r_{m}^{\sigma} e^{-r_{m}^{\sigma} y}\right\} \\
& \times\left(\int_{-b}^{b} \sigma(\xi) e^{i \pi(m-\varepsilon) \xi / a} d \xi\right) e^{-i \pi(m-\varepsilon) x / a}, \quad y \geq 0, \\
& u_{y}(x, y)=\frac{1}{2 a \mu} \sum_{m=-\infty}^{+\infty} \frac{q_{m}^{\sigma}}{\Delta_{m}}\left\{\left[k_{2}^{2}-2\left[\frac{\pi(m-\varepsilon)}{a}\right]^{2}\right] e^{-q_{m}^{\sigma} y}+2\left[\frac{\pi(m-\varepsilon)}{a}\right]^{2} e^{-r_{m}^{\sigma} y}\right\} \\
& \times\left(\int_{-b}^{b} \sigma(\xi) e^{i \pi(m-\varepsilon) \xi / a} d \xi\right) e^{-i \pi(m-\varepsilon) x / a}, \quad y \geq 0
\end{aligned}
$$


where the kernel $K^{\sigma}$ is given by

$$
K^{\sigma}(x)=\sum_{m=-\infty}^{+\infty} \frac{q_{m}^{\sigma}}{a \Delta_{m}} e^{-i \pi(m-\varepsilon) x / a},
$$

and we put

$$
\begin{aligned}
C^{\sigma} & =2 \mu u_{0} / k_{2}^{2}, \quad q_{m}^{\sigma}=\sqrt{[\pi(m-\varepsilon) / a]^{2}-k_{1}^{2}}, \quad r_{m}^{\sigma}=\sqrt{[\pi(m-\varepsilon) / a]^{2}-k_{2}^{2}}, \\
\Delta_{m} & =\Delta(\pi(m-\varepsilon) / a)=\left\{2[\pi(m-\varepsilon) / a]^{2}-k_{2}^{2}\right\}^{2}-4[\pi(m-\varepsilon) / a]^{2} q_{m}^{\sigma} r_{m}^{\sigma} .
\end{aligned}
$$

When real, the root-squares in $(3.5 \mathrm{~b}),(3.8 \mathrm{~b})$ will be taken as positive.

4. Approximation for explicit solutions. Of course, the above integral equations could be directly submitted to classical numerical algorithms for arbitrary values of the involved parameters (and actually this has been done for the sake of comparison). However, in this paper we aim to remain in an analytical context, and to this end we assume that frequency belongs to some specific ranges according to the value of the phase shift $\varepsilon \in(0,1) 11$ In this connection, for each one of the two problems being considered, we shall treat separately the three cases (1) $\varepsilon<1 / 2$, (2) $\varepsilon>1 / 2,(3) \varepsilon=1 / 2$, which may have different physical implications.

4.1. Anti-plane problem.

4.1.1. The case $\varepsilon<1 / 2$. Let us put

$$
a k / \pi<1-\varepsilon
$$

looking at (3.5), we accept that, as a consequence, the kernel in the integral equation (3.3) can be simplified by means of the approximation

$$
a q_{m}^{\tau} \simeq \pi|m-\varepsilon| \quad \forall m \neq 0 .
$$

It is worth remarking that this is not a low-frequency approximation, since, for example, at one third of the range $(4.1)$, it implies at worst (for $\left.q_{1}^{\tau}\right): \sqrt{1-1 / 9}=0.94 \simeq 1$. The value of $q_{m}^{\tau}$ for $m=0$ is taken to be exact. In this connection, we note that the wave number $q_{0}^{\tau}$ is imaginary for $a k / \pi$ in the range $(\varepsilon, 1-\varepsilon)$, being positive for $a k / \pi$ less than $\varepsilon$. Equation (3.4) thus implies non-decaying (far-field) wave propagation (of the mode with order zero) only in that range (of course, for $a k / \pi>1-\varepsilon$ further propagating modes arise).

Thus, from (3.5a) we get

$$
\begin{aligned}
K^{\tau}(x-\xi) & =\sum_{m=-\infty}^{\infty} \frac{1}{a q_{m}^{\tau}} e^{-i \pi(m-\varepsilon)(x-\xi) / a} \\
& =e^{i \pi \varepsilon(x-\xi) / a} \times\left[\frac{1}{a q_{0}^{\tau}}+\sum_{m \neq 0} \frac{1}{a q_{m}^{\tau}} e^{-i \pi m(x-\xi) / a}\right] \\
& =e^{i \pi \varepsilon(x-\xi) / a} \times\left[\frac{1}{a q_{0}^{\tau}}+\frac{1}{\pi} K_{\varepsilon}(x-\xi)\right],
\end{aligned}
$$

\footnotetext{
${ }^{1}$ The case $\varepsilon=0$, in which all punches vibrate with the same phase, has been treated in [7.
} 
where

$$
K_{\varepsilon}(x) \equiv \sum_{m \neq 0} \frac{e^{-i \pi m x / a}}{|m-\varepsilon|}
$$

is a kernel independent of frequency.

By introducing the new unknown

$$
\varphi_{\varepsilon}(\xi) \equiv e^{-i \pi \varepsilon \xi / a} \tau(\xi),
$$

it is not difficult to deduce, starting from (3.3), the following integral equation for such a function $(|x|<b)$ :

$$
\frac{1}{\pi} \int_{-b}^{b} K_{\varepsilon}(x-\xi) \varphi_{\varepsilon}(\xi) d \xi=C^{\tau} e^{-i \pi \varepsilon x / a}-\frac{1}{a q_{0}^{\tau}} \Phi_{\varepsilon},
$$

where

$$
\Phi_{\varepsilon}=\int_{-b}^{b} \varphi_{\varepsilon}(\xi) d \xi
$$

is an unknown constant.

Now, it is clear that, if $h_{0}(\xi), h_{\varepsilon}(\xi)$ are two functions which solve the auxiliary integral equations

$$
\frac{1}{\pi} \int_{-b}^{b} K_{\varepsilon}(x-\xi)\left(\begin{array}{c}
h_{0}(\xi) \\
h_{\varepsilon}(\xi)
\end{array}\right) d \xi=\left(\begin{array}{l}
1 \\
e^{-i \pi \varepsilon x / a}
\end{array}\right), \quad x \in(-b, b)
$$

fully independent of frequency, then, by linearity, the solution of (4.6) can be constructed as

$$
\varphi_{\varepsilon}(x)=-\frac{1}{a q_{0}^{\tau}} \Phi_{\varepsilon} h_{0}(x)+C^{\tau} h_{\varepsilon}(x)
$$

from which $\tau(x)$ is rapidly deduced 2 The unknown constant above can be obtained by integrating $(4.8)$ in $(-b, b)$; we get

$$
\Phi_{\varepsilon}=\left(1+\frac{1}{a q_{0}^{\tau}} H_{0}\right)^{-1} C^{\tau} H_{\varepsilon}
$$

where new constants $H$ (free of frequency) are given by

$$
H_{0}=\int_{-b}^{b} h_{0}(x) d x, \quad H_{\varepsilon}=\int_{-b}^{b} h_{\varepsilon}(x) d x
$$

and can be calculated after the equations (4.7) have been solved.

4.1.2. The case $\varepsilon>1 / 2$. Let us put

$$
a k / \pi<\varepsilon ;
$$

looking at (3.5a) and (3.5b), we accept that, as a consequence, the kernel can be simplified by the approximation

$$
a q_{m}^{\tau} \simeq \pi|m-\varepsilon| \quad \forall m \neq 1 .
$$

A remark similar to that after (4.2) applies (for $q_{0}^{\tau}$ ). The value of $q_{m}^{\tau}$ for $m=1$ is taken to be exact. In this connection, note that the wave number $q_{1}^{\tau}$ is imaginary for $a k / \pi$ in the

\footnotetext{
${ }^{2}$ Note however that in the integral of (3.4), the function $\varphi$ can directly appear.
} 
range $(1-\varepsilon, \varepsilon)$, being positive for $a k / \pi$ less than $1-\varepsilon$. Equation (3.4) thus implies farfield propagation (of the mode with order 1) only in that range (of course, for $a k / \pi>\varepsilon$, further propagating modes arise).

Thus, from (3.5a) we get

$$
\begin{aligned}
K^{\tau}(x-\xi) & =\sum_{m=-\infty}^{\infty} \frac{1}{a q_{m}^{\tau}} e^{-i \pi(m-\varepsilon)(x-\xi) / a} \\
& =e^{i \pi(\varepsilon-1)(x-\xi) / a} \times\left[\frac{1}{a q_{1}^{\tau}}+\sum_{m \neq 1} \frac{1}{a q_{m}^{\tau}} e^{-i \pi(m-1)(x-\xi) / a}\right] \\
& =e^{i \pi(\varepsilon-1)(x-\xi) / a} \times\left[\frac{1}{a q_{1}^{\tau}}+\frac{1}{\pi} K_{\varepsilon}^{\prime}(x-\xi)\right]
\end{aligned}
$$

where

$$
K_{\varepsilon}^{\prime}(x) \equiv \sum_{m \neq 1} \frac{e^{-i \pi(m-1) x / a}}{|m-\varepsilon|}=\sum_{m \neq 0} \frac{e^{-i \pi m x / a}}{|m-(\varepsilon-1)|} \equiv K_{\varepsilon-1}(x)
$$

is a kernel independent of frequency. It is now clear that the subsequent development of this case can be obtained from the previous one by the only substitution $\varepsilon \rightarrow \varepsilon-1$ in all formulas (after (4.4)). In this connection, we will denote by $\hat{h}_{0}(\xi)$ and $\hat{h}_{\varepsilon-1}(\xi)$ the solutions of the auxiliary equations (4.7) with $\varepsilon-1$ instead of $\varepsilon$ (in the kernel and right-hand side), and by $\hat{H}_{0}, \hat{H}_{\varepsilon-1}$ the corresponding integrals over $(-b, b)$.

4.1.3. The case $\varepsilon=1 / 2$. This is a more particular case, when each pair of adjacent punches vibrates in anti-phase. Let us put

$$
a k / \pi<3 / 2
$$

looking at (3.5a) and (3.5b), we accept that, as a consequence, the kernel can be simplified by the approximation

$$
a q_{m}^{\tau} \simeq \pi|m-1 / 2| \quad \forall m \neq 0,1 .
$$

A remark similar to that after (4.2) applies (for $q_{2}^{\tau}=q_{-1}^{\tau}$ ). The value of $q_{m}^{\tau}$ for $m=0,1$ is taken to be exact. In this connection, note that the wave number $q_{0}^{\tau}\left(=q_{1}^{\tau}\right)$ is imaginary for $a k / \pi$ in the range $(1 / 2,3 / 2)$, being positive for $a k / \pi$ less than $1 / 2$. Equation (3.4) thus implies far-field propagation (of the modes with order 0,1 which have the same dependence on $y$ ) only in that range (of course, for $a k / \pi>3 / 2$ further propagating modes arise).

Thus, from (3.5a) we get

$$
\begin{aligned}
& K^{\tau}(x-\xi)=\sum_{m=-\infty}^{\infty} \frac{1}{a q_{m}^{\tau}} e^{-i \pi(m-1 / 2)(x-\xi) / a} \\
& =e^{i(\pi / 2)(x-\xi) / a} \times\left[\frac{1}{a q_{0}^{\tau}}\left(1+e^{-i \pi(x-\xi) / a}\right)+\sum_{m \neq 0,1} \frac{1}{a q_{m}^{\tau}} e^{-i \pi m(x-\xi) / a}\right] \\
& =e^{i(\pi / 2)(x-\xi) / a} \times\left[\frac{1}{a q_{0}^{\tau}}+\left(\frac{1}{a q_{0}^{\tau}}-\frac{2}{\pi}\right) e^{-i \pi(x-\xi) / a}+\frac{1}{\pi} K_{1 / 2}(x-\xi)\right]
\end{aligned}
$$


where

$$
K_{1 / 2}(x) \equiv \sum_{m \neq 0} \frac{e^{-i \pi m x / a}}{|m-1 / 2|}
$$

is a kernel independent of frequency.

By introducing the new unknowns,

$$
\varphi_{ \pm}(\xi) \equiv e^{ \pm i(\pi / 2) \xi / a} \tau(\xi)
$$

it is not difficult to deduce, starting from (3.3), the following integral equation involving such functions $(|x|<b)$ :

$$
\begin{aligned}
\frac{1}{\pi} \int_{-b}^{b} K_{1 / 2}(x-\xi) \varphi_{-}(\xi) d \xi & =C^{\tau} e^{-i(\pi / 2) x / a} \\
& -\frac{1}{a q_{0}^{\tau}} \Phi_{-}+\left(\frac{2}{\pi}-\frac{1}{a q_{0}^{\tau}}\right) \Phi_{+} e^{-i \pi x / a}
\end{aligned}
$$

where

$$
\Phi_{ \pm}=\int_{-b}^{b} \varphi_{ \pm}(\xi) d \xi
$$

are unknown constants.

Now, it is clear that, if $\tilde{h}_{0}(\xi), \tilde{h}_{1 / 2}(\xi), \tilde{h}_{1}(\xi)$ are three functions which solve the auxiliary integral equations

$$
\frac{1}{\pi} \int_{-b}^{b} K_{1 / 2}(x-\xi)\left(\begin{array}{c}
\tilde{h}_{0}(\xi) \\
\tilde{h}_{1 / 2}(\xi) \\
\tilde{h}_{1}(\xi)
\end{array}\right) d \xi=\left(\begin{array}{c}
1 \\
e^{-i(\pi / 2) x / a} \\
e^{-i \pi x / a}
\end{array}\right), \quad x \in(-b, b),
$$

fully independent on frequency, then, by linearity, the solution of (4.20) can be constructed as

$$
\varphi_{-}(x)=-\frac{1}{a q_{0}^{\tau}} \Phi_{-} \tilde{h}_{0}(x)+C^{\tau} \tilde{h}_{1 / 2}(x)+\left(\frac{2}{\pi}-\frac{1}{a q_{0}^{\tau}}\right) \Phi_{+} \tilde{h}_{1}(x),
$$

from which $\tau(x)$ is rapidly deduced (recall footnote 2 ). The unknown constants $\Phi_{ \pm}$can be obtained by twice integrating $(4.22)$ in $(-b, b)$, as it is and after multiplying by $e^{i \pi x / a}$; we get an algebraic linear system, as follows:

$$
\left\{\begin{array}{c}
\left(1+\frac{1}{a q_{0}^{\tau}} \tilde{H}_{0}\right) \Phi_{-}+\left(\frac{1}{a q_{0}^{\tau}}-\frac{2}{\pi}\right) \tilde{H}_{1} \Phi_{+}=C^{\tau} \tilde{H}_{1 / 2}, \\
\frac{1}{a q_{0}^{\tau}} \tilde{H}_{0}^{1} \Phi_{-}+\left[1+\left(\frac{1}{a q_{0}^{\tau}}-\frac{2}{\pi}\right) \tilde{H}_{1}^{1}\right] \Phi_{+}=C^{\tau} \tilde{H}_{1 / 2}^{1},
\end{array}\right.
$$

where new constants $\tilde{H}$ (free of frequency) are given by

$$
\tilde{H}_{\mu}^{\lambda}=\int_{-b}^{b} \tilde{h}_{\mu}(x) e^{i \lambda \pi x / a} d x \quad\left(\mu=0,1 / 2,1, \lambda=0,1 ; \tilde{H}_{\mu}^{0} \equiv \tilde{H}_{\mu}\right)
$$

and can be calculated after the equations (4.21) have been solved. 


\subsection{In-plane problem.}

4.2.1. The case $\varepsilon<1 / 2$. Let us put

$$
a k_{2} / \pi<1-\varepsilon
$$

looking at (3.8a) and (3.8b), we accept that, as a consequence, the kernel in the integral equation (3.6) can be simplified by means of the approximations

$$
a q_{m}^{\sigma} \simeq a r_{m}^{\sigma} \simeq \pi|m-\varepsilon|, \quad \Delta_{m} \simeq 2\left(\frac{\pi(m-\varepsilon)}{a}\right)^{2}\left(k_{1}^{2}-k_{2}^{2}\right) \quad \forall m \neq 0 .
$$

A remark similar to that after (4.2) applies (for $r_{1}^{\sigma}$ ). The values of all parameters above for $m=0$ are taken to be exact. In this connection, we note that the wave number $r_{0}^{\sigma}$ is imaginary for $a k_{2} / \pi$ in the range $(\varepsilon, 1-\varepsilon)$, being positive, along with $q_{0}^{\sigma}$, for $a k_{2} / \pi$ less than $\varepsilon$. Equations (3.7a) and (3.7b) thus imply far-field propagation (of the mode with order zero) only in that range (of course, for $a k_{2} / \pi>1-\varepsilon$ further propagating modes arise).

Thus, from (3.8a) we get

$$
\begin{aligned}
K^{\sigma}(x-\xi) & =\sum_{m=-\infty}^{\infty} \frac{q_{m}^{\sigma}}{a \Delta_{m}} e^{-i \pi(m-\varepsilon)(x-\xi) / a} \\
& =e^{i \pi \varepsilon(x-\xi) / a} \times\left[\frac{q_{0}^{\sigma}}{a \Delta_{0}}+\sum_{m \neq 0} \frac{q_{m}^{\sigma}}{a \Delta_{m}} e^{-i \pi m(x-\xi) / a}\right] \\
& =e^{i \pi \varepsilon(x-\xi) / a} \times\left[\frac{q_{0}^{\sigma}}{a \Delta_{0}}+\frac{1 /(2 \pi)}{k_{1}^{2}-k_{2}^{2}} K_{\varepsilon}(x-\xi)\right],
\end{aligned}
$$

where $K_{\varepsilon}(x)$ (free of frequency) is given by (4.4). The subsequent development is analogous to that shown for the anti-plane problem in this case: Equations (4.5), (4.6a), (4.6b), (4.8) and (4.9) are respectively replaced by:

$$
\begin{gathered}
\psi_{\varepsilon}(\xi) \equiv e^{-i \pi \varepsilon \xi / a} \sigma(\xi), \\
\frac{1}{\pi} \int_{-b}^{b} K_{\varepsilon}(x-\xi) \psi_{\varepsilon}(\xi) d \xi=2\left(k_{1}^{2}-k_{2}^{2}\right) C^{\sigma} e^{-i \pi \varepsilon x / a}-2\left(k_{1}^{2}-k_{2}^{2}\right) \frac{q_{0}^{\sigma}}{a \Delta_{0}} \Psi_{\varepsilon}, \\
\Psi_{\varepsilon}=\int_{-b}^{b} \psi_{\varepsilon}(\xi) d \xi \\
\psi_{\varepsilon}(x)=-2\left(k_{1}^{2}-k_{2}^{2}\right) \frac{q_{0}^{\sigma}}{a \Delta_{0}} \Psi_{\varepsilon} h_{0}(x)+2\left(k_{1}^{2}-k_{2}^{2}\right) C^{\sigma} h_{\varepsilon}(x), \\
\Psi_{\varepsilon}=\left[\frac{1}{2\left(k_{1}^{2}-k_{2}^{2}\right)}+\frac{q_{0}^{\sigma}}{a \Delta_{0}} H_{0}\right]^{-1} C^{\sigma} H_{\varepsilon} .
\end{gathered}
$$

(Of course, (4.7) and (4.10) apply unaltered.)

4.2.2. The case $\varepsilon>1 / 2$. Let us put

$$
a k_{2} / \pi<\varepsilon
$$


looking at (3.8a) and (3.8b), we accept that, as a consequence, the kernel can be simplified by the approximations

$$
a q_{m}^{\sigma} \simeq a r_{m}^{\sigma} \simeq \pi|m-\varepsilon|, \quad \Delta_{m} \simeq 2\left(\frac{\pi(m-\varepsilon)}{a}\right)^{2}\left(k_{1}^{2}-k_{2}^{2}\right) \quad \forall m \neq 1 .
$$

A remark similar to that after (4.2) applies (for $r_{0}^{\sigma}$ ). The values of all parameters above for $m=1$ are taken to be exact. In this connection, note that the wave number $r_{1}^{\sigma}$ is imaginary for $a k_{2} / \pi$ in the range $(1-\varepsilon, \varepsilon)$, being positive, along with $q_{1}^{\sigma}$, for $a k_{2} / \pi$ less than $1-\varepsilon$. Equations (3.7a) and (3.7b) thus imply far-field propagation (of the mode with order 1) only in that range (of course, for $a k_{2} / \pi>\varepsilon$ further propagating modes arise).

Thus, from (3.8a) we get

$$
\begin{aligned}
K^{\sigma}(x-\xi) & =\sum_{m=-\infty}^{\infty} \frac{q_{m}^{\sigma}}{a \Delta_{m}} e^{-i \pi(m-\varepsilon)(x-\xi) / a} \\
& =e^{i \pi(\varepsilon-1)(x-\xi) / a} \times\left[\frac{q_{1}^{\sigma}}{a \Delta_{1}}+\sum_{m \neq 1} \frac{q_{m}^{\sigma}}{a \Delta_{m}} e^{-i \pi(m-1)(x-\xi) / a}\right] \\
& =e^{i \pi(\varepsilon-1)(x-\xi) / a} \times\left[\frac{q_{1}^{\sigma}}{a \Delta_{1}}+\frac{1 /(2 \pi)}{k_{1}^{2}-k_{2}^{2}} K_{\varepsilon}^{\prime}(x-\xi)\right],
\end{aligned}
$$

where $K_{\varepsilon}^{\prime}(x)=K_{\varepsilon-1}(x)$ (free of frequency) is given by (4.14). It is now clear that the subsequent development of this case can be obtained from the previous one by the only substitution $\varepsilon \rightarrow \varepsilon-1$ in all formulas (4.28)-(4.31) (along with the equations (4.7), for which recall the final remark of subsection 4.1.2).

4.2.3. The case $\varepsilon=1 / 2$. As already noted, this is a more particular case (when each pair of adjacent punches vibrates in anti-phase). Let us put

$$
a k_{2} / \pi<3 / 2
$$

looking at (3.8a) and (3.8b), we accept that, as a consequence, the kernel can be simplified by the approximations

$$
a q_{m}^{\sigma} \simeq a r_{m}^{\sigma} \simeq \pi|m-1 / 2|, \quad \Delta_{m} \simeq 2\left(\frac{\pi(m-1 / 2)}{a}\right)^{2}\left(k_{1}^{2}-k_{2}^{2}\right) \quad \forall m \neq 0,1 .
$$

A remark similar to that after (4.2) applies (for $r_{2}^{\sigma}=r_{-1}^{\sigma}$ ). The values of all parameters above for $m=0,1$ are taken to be exact. In this connection, note that the wave number $r_{0}^{\sigma}\left(=r_{1}^{\sigma}\right)$ is imaginary for $a k_{2} / \pi$ in the range $(1 / 2,3 / 2)$, being positive, along with $q_{0}^{\sigma}(=$ $\left.q_{1}^{\sigma}\right)$, for $a k_{2} / \pi$ less than $1 / 2$. Equations (3.7a) and (3.7b) thus imply far-field propagation (of the modes with order 0,1 which have the same dependence on $y$ ) only in that range (of course, for $a k_{2} / \pi>3 / 2$ further propagating modes arise). 
Thus, from (3.8a) we get

$$
\begin{aligned}
& K^{\sigma}(x-\xi)=\sum_{m=-\infty}^{\infty} \frac{q_{m}^{\sigma}}{a \Delta_{m}} e^{-i \pi(m-1 / 2)(x-\xi) / a} \\
& =e^{i(\pi / 2)(x-\xi) / a} \times\left[\frac{q_{0}^{\sigma}}{a \Delta_{0}}\left(1+e^{-i \pi(x-\xi) / a}\right)+\sum_{m \neq 0,1} \frac{q_{m}^{\sigma}}{a \Delta_{m}} e^{-i \pi m(x-\xi) / a}\right] \\
& =e^{i(\pi / 2)(x-\xi) / a} \times\left[\frac{q_{0}^{\sigma}}{a \Delta_{0}}+\left(\frac{q_{0}^{\sigma}}{a \Delta_{0}}-\frac{1 / \pi}{k_{1}^{2}-k_{2}^{2}}\right) e^{-i \pi(x-\xi) / a}+\frac{1 /(2 \pi)}{k_{1}^{2}-k_{2}^{2}} K_{1 / 2}(x-\xi)\right],
\end{aligned}
$$

where $K_{1 / 2}(x)$ (free of frequency) is given by (4.18). The subsequent development is analogous to that shown for the anti-plane problem in this case: Equations (4.19), (4.20a), $(4.20 \mathrm{~b}),(4.22)$ and $(4.23)$ are respectively replaced by:

$$
\begin{gathered}
\psi_{ \pm}(\xi) \equiv e^{ \pm i(\pi / 2) \xi / a} \sigma(\xi) \\
\frac{1}{\pi} \int_{-b}^{b} K_{1 / 2}(x-\xi) \psi_{-}(\xi) d \xi=2\left(k_{1}^{2}-k_{2}^{2}\right) C^{\sigma} e^{-i(\pi / 2) x / a} \\
-2\left(k_{1}^{2}-k_{2}^{2}\right) \frac{q_{0}^{\sigma}}{a \Delta_{0}} \Psi_{-}+\left[\frac{2}{\pi}-2\left(k_{1}^{2}-k_{2}^{2}\right) \frac{q_{0}^{\sigma}}{a \Delta_{0}}\right] \Psi_{+} e^{-i \pi x / a} \\
\Psi_{ \pm}=\int_{-b}^{b} \psi_{ \pm}(\xi) d \xi \\
\psi_{-}(x)=-2\left(k_{1}^{2}-k_{2}^{2}\right) \frac{q_{0}^{\sigma}}{a \Delta_{0}} \Psi_{-} \tilde{h}_{0}(x)+2\left(k_{1}^{2}-k_{2}^{2}\right) C^{\sigma} \tilde{h}_{1 / 2}(x) \\
+\left[\frac{2}{\pi}-2\left(k_{1}^{2}-k_{2}^{2}\right) \frac{q_{0}^{\sigma}}{a \Delta_{0}}\right] \Psi_{+} \tilde{h}_{1}(x) \\
\left\{\begin{array}{c}
{\left[1+2\left(k_{1}^{2}-k_{2}^{2}\right) \frac{q_{0}^{\sigma}}{a \Delta_{0}} \tilde{H}_{0}\right] \Psi_{-}+\left[2\left(k_{1}^{2}-k_{2}^{2}\right) \frac{q_{0}^{\sigma}}{a \Delta_{0}}-\frac{2}{\pi}\right] \tilde{H}_{1} \Psi_{+}=2\left(k_{1}^{2}-k_{2}^{2}\right) C^{\sigma} \tilde{H}_{1 / 2}} \\
2\left(k_{1}^{2}-k_{2}^{2}\right) \frac{q_{0}^{\sigma}}{a \Delta_{0}} \tilde{H}_{0}^{1} \Psi_{-}+\left\{1+\left[2\left(k_{1}^{2}-k_{2}^{2}\right) \frac{q_{0}^{\sigma}}{a \Delta_{0}}-\frac{2}{\pi}\right] \tilde{H}_{1}^{1}\right\} \Psi_{+}=2\left(k_{1}^{2}-k_{2}^{2}\right) C^{\sigma} \tilde{H}_{1 / 2}^{1} .
\end{array}\right.
\end{gathered}
$$

(Of course, (4.21) and (4.24) apply unaltered.)

5. Analytical treatment of the basic integral equations. Let us rewrite all the auxiliary integral equations of the previous section in the general form:

$$
\frac{1}{\pi} \int_{-b}^{b} K_{\eta}(x-\xi) h(\xi) d \xi=f(x), \quad|x| \leq b
$$

where

$$
K_{\eta}(x)=\sum_{m \neq 0} \frac{e^{-i \pi m x / a}}{|m-\eta|} .
$$

By $\eta$ we mean $\varepsilon$ when it is $\varepsilon \leq 1 / 2$, or $\varepsilon-1$ when $\varepsilon>1 / 2$; so, $\eta$ can be negative, but its modulus never exceeds $1 / 2$. Of course, $f(x)$ and $h(\xi)$ are also intended to depend on the parameter $\eta$. 
By applying the operator $D(\cdot)=-(a / i \pi) d / d x(\cdot)-\eta I(\cdot)$, where $I$ is the identical operator, (5.1a) and (5.1b) become

$$
\int_{-b}^{b} \sum_{m \neq 0} \operatorname{sign}(m-\varepsilon) e^{-i \pi m(x-\xi) / a} h(\xi) d \xi=(D f)(x), \quad|x| \leq b ;
$$

$\operatorname{since} \operatorname{sign}(m-\eta)=\operatorname{sign} m$, we have

$$
\begin{aligned}
\sum_{m \neq 0} \operatorname{sign}(m-\eta) e^{-i \pi m(x-\xi) / a} & =\sum_{m=1}^{\infty} e^{-i \pi m(x-\xi) / a}-\sum_{m=1}^{\infty} e^{i \pi m(x-\xi) / a} \\
& =\frac{e^{i \pi x / a}+e^{i \pi \xi / a}}{e^{i \pi x / a}-e^{i \pi \xi / a}}
\end{aligned}
$$

By the change of variables

$$
z=e^{i \pi x / a}, \quad \tau=e^{i \pi \xi / a}, \quad d \xi=\frac{a}{i \pi} \frac{d \tau}{\tau}
$$

(5.2) is transformed into

$$
\frac{a}{i \pi} \int_{\alpha}^{\beta} \frac{h(\tau)}{\tau} \frac{z+\tau}{z-\tau} d \tau=\pi(D f)(z), \quad z \in L(\alpha, \beta),
$$

where $\alpha=\exp (-i \pi b / a), \beta=\exp (i \pi b / a)$, the contour $L(\alpha, \beta)=\left\{z=e^{i \phi}:|\phi|<\pi b / a\right\}$ is an open part of the unit circle in the complex plane, and the symbol $\int_{\alpha}^{\beta}(\cdot)$ means (henceforth) the integral over such a contour. For simplicity, we use the same symbol for the functions $h$ and $D f$ when passing to the complex variables.

Since $\frac{z+\tau}{(z-\tau) \tau}=\frac{1}{\tau}+\frac{2}{z-\tau},(5.5)$ is equivalent to the following Cauchy-type singular integral equation:

$$
\frac{1}{i \pi} \int_{\alpha}^{\beta} \frac{h(\tau)}{\tau-z} d \tau=\frac{H}{2 a}-\frac{\pi}{2 a}(D f)(z) \equiv F(z), \quad z \in L(\alpha, \beta)
$$

where

$$
H=\frac{a}{i \pi} \int_{\alpha}^{\beta} \frac{h(\tau)}{\tau} d \tau=\int_{-b}^{b} h(\xi) d \xi
$$

is a (still unknown) constant.

The solution to (5.6a) and (5.6b) can be given as [10]

$$
h(z)=\frac{1}{i \pi \sqrt{(z-\alpha)(\beta-z)}}\left[C+\int_{\alpha}^{\beta} \frac{F(\tau) \sqrt{(\tau-\alpha)(\beta-\tau)}}{\tau-z} d \tau\right], \quad z \in L(\alpha, \beta),
$$

where $C$ is an arbitrary constant.

Now, recalling the right-hand terms in the auxiliary equations, let us firstly put $f(x)=$ 1. This trivially implies that

$$
(D f)(x)=(D f)(z)=-\eta \quad \text { and } \quad F(z)=\frac{H+\pi \eta}{2 a},
$$


so that the integral in brackets above holds in this case:

$$
\begin{gathered}
\int_{\alpha}^{\beta} \frac{F(\tau) \sqrt{(\tau-\alpha)(\beta-\tau)}}{\tau-z} d \tau=\frac{H+\pi \eta}{2 a} \int_{\alpha}^{\beta} \frac{\sqrt{(\tau-\alpha)(\beta-\tau)}}{\tau-z} d \tau \\
=\frac{H+\pi \eta}{2 a} \pi\left(\frac{\alpha+\beta}{2}-z\right), \quad z \in L(\alpha, \beta),
\end{gathered}
$$

where the tabular integral 9 ]

$$
\int_{\alpha}^{\beta} \frac{\sqrt{(\tau-\alpha)(\beta-\tau)}}{\tau-z} d \tau=\pi\left(\frac{\alpha+\beta}{2}-z\right)
$$

has been used. Hence, solution (5.7) becomes

$$
h(z)=\frac{C+\pi(\alpha+\beta)(H+\pi \eta) /(4 a)}{i \pi \sqrt{(z-\alpha)(\beta-z)}}-\frac{(H+\pi \eta) z}{2 a i \sqrt{(z-\alpha)(\beta-z)}}, \quad z \in L(\alpha, \beta),
$$

in which two constants ( $H$ and $C$ ) remain to be determined. Substitution of $h$ from (5.10) into (5.6b) gives a first relation between such constants, as follows:

$$
\begin{aligned}
H & =\frac{a C+\pi(\alpha+\beta)(H+\pi \eta) / 4}{-\pi^{2}} \int_{\alpha}^{\beta} \frac{d z}{z \sqrt{(z-\alpha)(\beta-z)}}+\frac{H+\pi \eta}{2 \pi} \int_{\alpha}^{\beta} \frac{d z}{\sqrt{(z-\alpha)(\beta-z)}} \\
& =-a C / \pi-(H+\pi \eta)(\alpha+\beta-2) / 4
\end{aligned}
$$

where the tabular integrals 9 ]

$$
\begin{array}{r}
J_{k}=\int_{\alpha}^{\beta} \frac{z^{k} d z}{\sqrt{(z-\alpha)(\beta-z)}}=\pi^{2}(-1)^{k} \sum_{\ell=0}^{k} \frac{\alpha^{\ell} \beta^{k-\ell}}{\ell !(k-\ell) ! \Gamma\left(\frac{1}{2}-\ell\right) \Gamma\left(\frac{1}{2}+\ell-k\right)}, \\
J_{-k}=J_{k-1}, \quad k=0,1,2, \ldots
\end{array}
$$

have been used (here for only $k=0,-1: J_{0}=J_{-1}=\pi ; \Gamma(z) \equiv \int_{0}^{\infty} e^{-t} t^{z-1} d t$ is the (Euler's) Gamma function).

Another relation can be obtained by calculating the original integral equation (5.1) at any particular value of $x$ in $(-b, b)$ (for instance, at $x=0$ ), then passing to the complex variables (5.4) and finally substituting $h$ from (5.10). We get

$$
\begin{aligned}
& \frac{a}{i \pi^{2}} \sum_{m \neq 0} \frac{1}{|m-\eta|} \int_{\alpha}^{\beta} \tau^{m-1} h(\tau) d \tau \\
& =\frac{a}{i \pi^{2}} \sum_{m \neq 0} \frac{1}{|m-\eta|}\left\{\left[\frac{C+\pi(\alpha+\beta)(H+\pi \eta) /(4 a)}{i \pi}\right] J_{m-1}-\frac{(H+\pi \eta)}{2 a i} J_{m}\right\}=1
\end{aligned}
$$

on putting 


$$
S_{1}(\eta)=\sum_{m \neq 0} \frac{J_{m-1}}{|m-\eta|}, \quad S_{2}(\eta)=\sum_{m \neq 0} \frac{J_{m}}{|m-\eta|}, 3
$$

we finally have

$$
\left[\frac{a C}{\pi}+\frac{H+\pi \eta}{4}(\alpha+\beta)\right] S_{1}(\eta)=\frac{H+\pi \eta}{2} S_{2}(\eta)-\pi^{2} .
$$

Solving the linear system (5.11), (5.15) gives the following expressions:

$$
H=\frac{2 \pi^{2}+\pi \eta\left[S_{1}(\eta)-S_{2}(\eta)\right]}{S_{1}(\eta)+S_{2}(\eta)}, \quad \frac{a C}{\pi}=\frac{\pi \eta}{4}(2-\alpha-\beta)-\frac{H}{4}(2+\alpha+\beta),
$$

by means of which (5.10) is fully explicit.

Let us now put $f(x)=\exp (-i \pi \eta x / a) \quad(\eta=\varepsilon \leq 1 / 2$ or $\eta=\varepsilon-1, \varepsilon>1 / 2)$. It follows that

$$
(D f)(x)=(D f)(z)=0 \quad \text { and } \quad F(z)=\frac{H}{2 a}
$$

so that

$$
\int_{\alpha}^{\beta} \frac{F(\tau) \sqrt{(\tau-\alpha)(\beta-\tau)}}{\tau-z} d \tau=\frac{\pi H}{2 a}\left(\frac{\alpha+\beta}{2}-z\right), \quad z \in L(\alpha, \beta) .
$$

As a consequence, (5.10) and (5.16) are replaced by

$$
\begin{gathered}
h(z)=\frac{C+\pi(\alpha+\beta) H /(4 a)}{i \pi \sqrt{(z-\alpha)(\beta-z)}}-\frac{H z}{2 a i \sqrt{(z-\alpha)(\beta-z)}}, \quad z \in L(\alpha, \beta) ; \\
H=\frac{2 \pi^{2}}{S_{1}(\eta)+S_{2}(\eta)}, \quad \frac{a C}{\pi}=-\frac{H}{4}(2+\alpha+\beta) .
\end{gathered}
$$

Finally, let us put $f(x)=\exp (-i \pi x / a)$, which is the last right-hand term to be concerned with for $\eta=1 / 2$ (see $\left.(4.21)_{3}\right)$. It follows that

$$
(D f)(x)=\frac{1}{2} e^{-i \pi x / a}, \quad(D f)(z)=\frac{1}{2 z} \quad \text { and } \quad F(z)=\frac{H}{2 a}-\frac{\pi}{4 a z},
$$

so that

$$
\begin{aligned}
\int_{\alpha}^{\beta} \frac{F(\tau) \sqrt{(\tau-\alpha)(\beta-\tau)}}{\tau-z} d \tau & =\frac{\pi H}{2 a}\left(\frac{\alpha+\beta}{2}-z\right)-\frac{\pi}{4 a} \int_{\alpha}^{\beta} \frac{\sqrt{(\tau-\alpha)(\beta-\tau)}}{\tau(\tau-z)} d \tau \\
& =\frac{\pi H}{2 a}\left(\frac{\alpha+\beta}{2}-z\right)-\frac{\pi^{2}}{4 a} \frac{1-z}{z}, \quad z \in L(\alpha, \beta),
\end{aligned}
$$

where the tabular integral 9

$$
\int_{\alpha}^{\beta} \frac{\sqrt{(\tau-\alpha)(\beta-\tau)}}{\tau} d \tau=\pi\left(\frac{\alpha+\beta}{2}-1\right)
$$

\footnotetext{
${ }^{3}$ Note that $S_{1,2}(-\eta)=S_{2,1}(\eta)$.
} 
has been used. As a consequence, the solution of $(4.21)_{3}$ is given (in the complex plane) by

$$
\begin{gathered}
\tilde{h}_{1}(x) \sim \tilde{h}_{1}(z)=\left\{\left[\frac{C}{i \pi}+\frac{H}{4 a i}(\alpha+\beta)+\frac{\pi}{4 a i}\right]-\frac{H}{2 a i} z-\frac{\pi}{4 a i} \frac{1}{z}\right\} \\
\begin{array}{c}
\frac{1}{\sqrt{(z-\alpha)(\beta-z)}} \\
z \in L(\alpha, \beta)
\end{array} \\
H=\frac{2 \pi^{2}-\frac{\pi}{2}\left[S_{3}(\eta)-\frac{\alpha+\beta}{2} S_{1}(\eta)\right]}{S_{1}(\eta)+S_{2}(\eta)} \equiv \tilde{H}_{1} \\
C=\frac{\pi}{a}\left[\frac{\pi}{4}\left(\frac{\alpha+\beta}{2}-1\right)-\left(\frac{\alpha+\beta}{4}+\frac{1}{2}\right) H\right] \equiv \tilde{C}_{1}
\end{gathered}
$$

where

$$
S_{3}(\eta)=\sum_{m \neq 0} \frac{J_{m-2}}{|m-\eta|}
$$

6. Explicit representations of the wave field. Recalling the terminology used in Section 4 , we can thus write

$$
\begin{gathered}
h_{0}(x) \sim h_{0}(z)=\frac{C+\pi(\alpha+\beta)(H+\pi \eta) /(4 a)}{i \pi \sqrt{(z-\alpha)(\beta-z)}}-\frac{(H+\pi \eta) z}{2 a i \sqrt{(z-\alpha)(\beta-z)}}, \quad z \in L(\alpha, \beta), \\
H=\frac{2 \pi^{2}+\pi \eta\left[S_{1}(\eta)-S_{2}(\eta)\right]}{S_{1}(\eta)+S_{2}(\eta)} \equiv H_{0} \\
C=\frac{\pi}{a}\left[\frac{\pi \eta}{4}(2-\alpha-\beta)-\frac{H}{4}(2+\alpha+\beta)\right] \equiv C_{0} \\
h_{\varepsilon}(x) \sim h_{\varepsilon}(z)=\frac{C+\pi(\alpha+\beta) H /(4 a)}{i \pi \sqrt{(z-\alpha)(\beta-z)}}-\frac{H z}{2 a i \sqrt{(z-\alpha)(\beta-z)}}, \quad z \in L(\alpha, \beta) \\
H=\frac{2 \pi^{2}}{S_{1}(\eta)+S_{2}(\eta)} \equiv H_{\varepsilon}, \quad C=-\frac{\pi}{a}\left[\frac{H}{4}(2+\alpha+\beta)\right] \equiv C_{\varepsilon}
\end{gathered}
$$

with $\eta=\varepsilon(\varepsilon<1 / 2)$ throughout where it appears on the right-hand sides.

$\hat{h}_{0}(x) \sim \hat{h}_{0}(z)$ and $\hat{h}_{\varepsilon-1}(x) \sim \hat{h}_{\varepsilon-1}(z)$ are equal to $h_{0}(z)$ and $h_{\varepsilon}(z)$ above, respectively, with $\eta=\varepsilon-1(\varepsilon>1 / 2)$ throughout where it appears; in this case, $H \equiv$ $\hat{H}_{0}, C \equiv \hat{C}_{0}$, and $H \equiv \hat{H}_{\varepsilon-1}, C \equiv \hat{C}_{\varepsilon-1}$, respectively.

$\tilde{h}_{0}(x) \sim \tilde{h}_{0}(z)$ and $\tilde{h}_{1 / 2}(x) \sim \tilde{h}_{1 / 2}(z)$ are equal to $h_{0}(z)$ and $h_{\varepsilon}(z)$ above, respectively, with $\eta=\varepsilon=1 / 2$ throughout where it appears; in this case, $H \equiv \tilde{H}_{0}, C \equiv$ $\tilde{C}_{0}$ and $H \equiv \tilde{H}_{1 / 2}, C \equiv \tilde{C}_{1 / 2}$, respectively.

$\tilde{h}_{1}(x) \sim \tilde{h}_{1}(z)$ is directly given by (5.23a) and (5.23b) (along with the corresponding constants $\left.\tilde{H}_{1}, \tilde{C}_{1}\right)$.

Now, let us go back to the representation formulas (3.4), (3.7) for the wave fields. In them, the integrals in brackets are calculated analytically by means of all previous formulas, as follows (we treat simultaneously both the anti-plane and in-plane problems). 
For $\varepsilon<1 / 2$, we have (starting from (4.8) and (4.30) and using (6.1) and (6.2)):

$$
\begin{gathered}
\int_{-b}^{b}\left(\begin{array}{c}
\tau(\xi) \\
\sigma(\xi)
\end{array}\right) e^{i \pi(m-\varepsilon) \xi / a} d \xi=\int_{-b}^{b}\left(\begin{array}{c}
\varphi_{\varepsilon}(\xi) \\
\psi_{\varepsilon}(\xi)
\end{array}\right) e^{i \pi m \xi / a} d \xi \\
=\left(\begin{array}{c}
-\frac{1}{a q_{0}^{\tau}} \Phi_{\varepsilon} \\
-2\left(k_{1}^{2}-k_{2}^{2}\right) \frac{q_{0}^{\sigma}}{a \Delta_{0}} \Psi_{\varepsilon}
\end{array}\right) \int_{-b}^{b} h_{0}(\xi) e^{i \pi m \xi / a} d \xi+\left(\begin{array}{c}
C^{\tau} \\
2\left(k_{1}^{2}-k_{2}^{2}\right) C^{\sigma}
\end{array}\right) \int_{-b}^{b} h_{\varepsilon}(\xi) e^{i \pi m \xi / a} d \xi \\
=\frac{a}{i \pi}\left(\begin{array}{c}
-\frac{1}{a q_{0}^{\tau}} \Phi_{\varepsilon} \\
-2\left(k_{1}^{2}-k_{2}^{2}\right) \frac{q_{0}^{\sigma}}{a \Delta_{0}} \Psi_{\varepsilon}
\end{array}\right) \int_{\alpha}^{\beta} h_{0}(\tau) \tau^{m-1} d \tau+\frac{a}{i \pi}\left(\begin{array}{c}
C^{\tau} \\
2\left(k_{1}^{2}-k_{2}^{2}\right) C^{\sigma}
\end{array}\right) \int_{\alpha}^{\beta} h_{\varepsilon}(\tau) \tau^{m-1} d \tau \\
=\frac{a}{i \pi}\left(\begin{array}{c}
-\frac{1}{a q_{0}^{\tau}} \Phi_{\varepsilon} \\
-2\left(k_{1}^{2}-k_{2}^{2}\right) \frac{q_{0}^{\sigma}}{a \Delta_{0}} \Psi_{\varepsilon}
\end{array}\right)\left[\frac{\pi \varepsilon}{2 a i}\left(J_{m-1}-J_{m}\right)-\frac{H_{0}}{2 a i}\left(J_{m-1}+J_{m}\right)\right] \\
+\frac{a}{i \pi}\left(\begin{array}{c}
C^{\tau} \\
2\left(k_{1}^{2}-k_{2}^{2}\right) C^{\sigma}
\end{array}\right)\left[-\frac{H_{\varepsilon}}{2 a i}\left(J_{m-1}+J_{m}\right)\right]
\end{gathered}
$$

where $\Phi_{\varepsilon}, \Psi_{\varepsilon}$ are given by (4.9) and (4.31). The propagating mode (of order $m=0$, when $a k / \pi$ or $a k_{2} / \pi>\varepsilon$ ) is simply given by

$$
\begin{gathered}
w_{\infty}(x, y)=-\frac{1}{2 a \mu q_{0}^{\tau}} e^{i \sqrt{k^{2}-(\pi \varepsilon / a)^{2}} y} \Phi_{\varepsilon} e^{i \pi \varepsilon x / a}, \quad y \rightarrow \infty ; \\
u_{x}^{\infty}(x, y)=-\frac{i \pi \varepsilon}{2 a^{2} \mu \Delta_{0}}\left\{\left[k_{2}^{2}-2\left(\frac{\pi \varepsilon}{a}\right)^{2}\right] e^{-q_{0}^{\sigma} y}+2 q_{0}^{\sigma} r_{0}^{\sigma} e^{i \sqrt{k_{2}^{2}-(\pi \varepsilon / a)^{2}} y}\right\} \Psi_{\varepsilon} e^{i \pi \varepsilon x / a}, \\
y \rightarrow \infty, \\
u_{y}^{\infty}(x, y)=\frac{q_{0}^{\sigma}}{2 a \mu \Delta_{0}}\left\{\left[k_{2}^{2}-2\left(\frac{\pi \varepsilon}{a}\right)^{2}\right] e^{-q_{0}^{\sigma} y}+2\left(\frac{\pi \varepsilon}{a}\right)^{2} e^{i \sqrt{k_{2}^{2}-(\pi \varepsilon / a)^{2}} y}\right\} \Psi_{\varepsilon} e^{i \pi \varepsilon x / a}, \\
y \rightarrow \infty .
\end{gathered}
$$

By a radiation condition [8], we took $q_{o}^{\tau}=-i \sqrt{k^{2}-(\pi \varepsilon / a)^{2}}, r_{o}^{\sigma}=-i \sqrt{k_{2}^{2}-(\pi \varepsilon / a)^{2}}$.

For $\varepsilon>1 / 2$, we have the same expressions as in (6.3) with the substitutions

$\varepsilon \rightarrow \varepsilon-1, C_{0} \rightarrow \hat{C}_{0}, H_{0} \rightarrow \hat{H}_{0}, C_{\varepsilon} \rightarrow \hat{C}_{\varepsilon-1}, H_{\varepsilon} \rightarrow \hat{H}_{\varepsilon-1}, J_{m-1} \rightarrow J_{m-2}, J_{m} \rightarrow J_{m-1}$.

The propagating mode (of order $m=1$, when $a k / \pi$ or $a k_{2} / \pi>1-\varepsilon$ ) is given by (6.4), (6.5a) and (6.5b) on simply putting $\varepsilon-1$ in place of $\varepsilon$ (and $\hat{H}_{0}, \hat{H}_{\varepsilon-1}$ in place of $H_{0}, H_{\varepsilon}$ in formulas (4.9) and (4.31)).

\footnotetext{
${ }^{4}$ Regarding $q_{0}^{\sigma}$, see (forthcoming) (7.3b), cases (II), (III).
} 
For $\varepsilon=1 / 2$, we have (starting from (4.22) and (4.40) and using (6.1), (6.2), and $(5.23))$ :

$$
\begin{aligned}
& \int_{-b}^{b}\left(\begin{array}{c}
\tau(\xi) \\
\sigma(\xi)
\end{array}\right) e^{i \pi(m-1 / 2) \xi / a} d \xi=\int_{-b}^{b}\left(\begin{array}{c}
\varphi_{-}(\xi) \\
\psi_{-}(\xi)
\end{array}\right) e^{i \pi m \xi / a} d \xi \\
& =\left(\begin{array}{c}
-\frac{1}{a q_{0}^{\tau}} \Phi_{-} \\
-2\left(k_{1}^{2}-k_{2}^{2}\right) \frac{q_{0}^{\sigma}}{a \Delta_{0}} \Psi_{-}
\end{array}\right) \int_{-b}^{b} \tilde{h}_{0}(\xi) e^{i \pi m \xi / a} d \xi+\left(\begin{array}{c}
C^{\tau} \\
2\left(k_{1}^{2}-k_{2}^{2}\right) C^{\sigma}
\end{array}\right) \int_{-b}^{b} \tilde{h}_{1 / 2}(\xi) e^{i \pi m \xi / a} d \xi \\
& -\left(\begin{array}{c}
\left(\frac{1}{a q_{0}^{\tau}}-\frac{2}{\pi}\right) \Phi_{+} \\
{\left[2\left(k_{1}^{2}-k_{2}^{2}\right) \frac{q_{0}^{\sigma}}{a \Delta_{0}}-\frac{2}{\pi}\right] \Psi_{+}}
\end{array}\right) \int_{-b}^{b} \tilde{h}_{1}(\xi) e^{i \pi m \xi / a} d \xi \\
& =\frac{a}{i \pi}\left(\begin{array}{c}
-\frac{1}{a q_{0}^{\tau}} \Phi_{-} \\
-2\left(k_{1}^{2}-k_{2}^{2}\right) \frac{q_{0}^{\sigma}}{a \Delta_{0}} \Psi_{-}
\end{array}\right) \int_{\alpha}^{\beta} \tilde{h}_{0}(\tau) \tau^{m-1} d \tau+\frac{a}{i \pi}\left(\begin{array}{c}
C^{\tau} \\
2\left(k_{1}^{2}-k_{2}^{2}\right) C^{\sigma}
\end{array}\right) \int_{\alpha}^{\beta} \tilde{h}_{1 / 2}(\tau) \tau^{m-1} d \tau \\
& -\frac{a}{i \pi}\left(\begin{array}{c}
\left(\frac{1}{a q_{0}^{\tau}}-\frac{2}{\pi}\right) \Phi_{+} \\
{\left[2\left(k_{1}^{2}-k_{2}^{2}\right) \frac{q_{0}^{\sigma}}{a \Delta_{0}}-\frac{2}{\pi}\right] \Psi_{+}}
\end{array}\right) \int_{\alpha}^{\beta} \tilde{h}_{1}(\tau) \tau^{m-1} d \tau \\
& =\frac{a}{i \pi}\left(\begin{array}{c}
-\frac{1}{a q_{0}^{\tau}} \Phi_{-} \\
-2\left(k_{1}^{2}-k_{2}^{2}\right) \frac{q_{0}^{\sigma}}{a \Delta_{0}} \Psi_{-}
\end{array}\right)\left[\frac{\pi / 2}{2 a i}\left(J_{m-1}-J_{m}\right)-\frac{\tilde{H}_{0}}{2 a i}\left(J_{m-1}+J_{m}\right)\right] \\
& +\frac{a}{i \pi}\left(\begin{array}{c}
C^{\tau} \\
2\left(k_{1}^{2}-k_{2}^{2}\right) C^{\sigma}
\end{array}\right)\left[-\frac{\tilde{H}_{1 / 2}}{2 a i}\left(J_{m-1}+J_{m}\right)\right] \\
& -\frac{a}{i \pi}\left(\begin{array}{c}
\left(\frac{1}{a q_{0}^{\tau}}-\frac{2}{\pi}\right) \Phi_{+} \\
{\left[2\left(k_{1}^{2}-k_{2}^{2}\right) \frac{q_{0}^{\sigma}}{a \Delta_{0}}-\frac{2}{\pi}\right] \Psi_{+}}
\end{array}\right)\left[\frac{\pi(\alpha+\beta) / 4-\tilde{H}_{1}}{2 a i} J_{m-1}-\frac{\tilde{H}_{1}}{2 a i} J_{m}-\frac{\pi}{4 a i} J_{m-2}\right],
\end{aligned}
$$


where $\Phi_{ \pm}, \Psi_{ \pm}$are given by systems (4.23) and (4.41), in which the constants $\tilde{H}_{0}, \tilde{H}_{1 / 2}, \tilde{H}_{1}$ are already known, while the remaining ones are calculated as follows:

$$
\begin{aligned}
\tilde{H}_{0}^{1}=\frac{a}{i \pi} \int_{\alpha}^{\beta} \tilde{h}_{0}(\tau) d \tau & =\frac{a}{i \pi}\left[\frac{\tilde{C}_{0}+\pi(\alpha+\beta)\left(\tilde{H}_{0}+\pi / 2\right) /(4 a)}{i \pi} J_{0}-\frac{\tilde{H}_{0}+\pi / 2}{2 a i} J_{1}\right] \\
& =-\frac{a}{\pi} \tilde{C}_{0}, \\
\tilde{H}_{1 / 2}^{1}=\frac{a}{i \pi} \int_{\alpha}^{\beta} \tilde{h}_{1 / 2}(\tau) d \tau & =\frac{a}{i \pi}\left[\frac{\tilde{C}_{1 / 2}+\pi(\alpha+\beta) \tilde{H}_{1 / 2} /(4 a)}{i \pi} J_{0}-\frac{\tilde{H}_{1 / 2}}{2 a i} J_{1}\right]=-\frac{a}{\pi} \tilde{C}_{1 / 2}, \\
\tilde{H}_{1}^{1}=\frac{a}{i \pi} \int_{\alpha}^{\beta} \tilde{h}_{1}(\tau) d \tau & =\frac{a}{i \pi}\left[\frac{\tilde{C}_{1}+\pi(\alpha+\beta) \tilde{H}_{1} /(4 a)+\pi^{2} /(4 a)}{i \pi} J_{0}-\frac{\tilde{H}_{1}}{2 a i} J_{1}-\frac{\pi}{4 a i} J_{-1}\right] \\
& =-\frac{a}{\pi} \tilde{C}_{1} .
\end{aligned}
$$

The propagating mode (of order $m=0,1$ when $a k / \pi$ or $a k_{2} / \pi>1 / 2$ ) is given by

$$
\begin{gathered}
w_{\infty}(x, y)=-\frac{1}{2 a \mu q_{0}^{\tau}} e^{i \sqrt{k^{2}-(\pi /(2 a))^{2}} y}\left(\Phi_{+} e^{-i \pi x /(2 a)}+\Phi_{-} e^{i \pi x /(2 a)}\right), \quad y \rightarrow \infty ; \\
u_{x}^{\infty}(x, y)=\frac{i \pi}{4 a^{2} \mu \Delta_{0}}\left\{\left[k_{2}^{2}-2\left(\frac{\pi}{2 a}\right)^{2}\right] e^{-q_{0}^{\sigma} y}+2 q_{0}^{\sigma} r_{0}^{\sigma} e^{i \sqrt{k_{2}^{2}-(\pi /(2 a))^{2}} y}\right\} \\
\times\left(\Psi_{+} e^{-i \pi x /(2 a)}-\Psi_{-} e^{i \pi x /(2 a)}\right), \quad y \rightarrow \infty, \\
u_{y}^{\infty}(x, y)=\frac{q_{0}^{\sigma}}{2 a \mu \Delta_{0}}\left\{\left[k_{2}^{2}-2\left(\frac{\pi}{2 a}\right)^{2}\right] e^{-q_{0}^{\sigma} y}+2\left(\frac{\pi}{2 a}\right)^{2} e^{i \sqrt{k_{2}^{2}-(\pi /(2 a))^{2}} y}\right\} \\
\times\left(\Psi_{+} e^{-i \pi x /(2 a)}+\Psi_{-} e^{i \pi x /(2 a)}\right), \quad y \rightarrow \infty
\end{gathered}
$$

(recall the remark after (6.5), along with the footnote).

As claimed, all the equations established in this section are clearly explicit with respect to frequency.

7. Physical remarks. As said in the Introduction, the case of a phase shift in the oscillation between adjacent punches implies very interesting (and rather specific) properties of the vibrating structure from the energy point of view. When all punches oscillate in the same phase, namely, when $\varepsilon=0$, propagating (non-decaying) waves are introduced into the elastic medium just from the lowest non-zero frequencies; see lines 3 in Figs. 2, 3 of [7]; physically, this means that some energy can be inputted throughout the medium even for arbitrarily small frequency.

On the contrary, when there is a certain phase shift, we see that all wave modes decay with distance for relatively low frequency, and this means that the energy can be deeply inputted only if the frequency is greater than a certain critical value (connected to the phase shift itself), starting from which a far-field propagation can occur; under such a value, the energy produced by the vibration remains concentrated in a zone of finite depth. The greatest critical value, namely, the wider range with no far-field propagation, 
corresponds to the anti-phase vibration $(\varepsilon=1 / 2)$. From a physical standpoint, this characteristic can be useful when one needs, for some practical applications, to concentrate the wave energy near the free surface of the medium; this happens, for example, in the detection of surface-breaking cracks by ultrasonic non-destructive testing. On the contrary, when one wishes to provide a high level of the wave energy far from the surface, like in the searching of mining deposits at large depths, higher frequencies should be applied.

Of course, by an obvious property of balance, the energy produced by the system of vibrating punches on the surface, averaged over the harmonic period, must be equal to the energy carried to infinity when there is far-field propagation, or to zero when there is no such propagation.

It is interesting to investigate these properties from a mathematical standpoint, in view of the analytical procedure here adopted. We can easily calculate the work made by the elastic stresses along the particles' displacements, during a period $2 \pi / \omega$ of the harmonic regime. After integrating over the typical interval $(-a, a)$, we get at infinity $($ as $y \rightarrow \infty)$ :

$$
E_{\infty}^{\tau} / \pi=\operatorname{Im}\left\{\int_{-a}^{a} \tau_{\infty}(x, y) w_{\infty}^{*}(x, y) d x\right\}
$$

or

$$
E_{\infty}^{\sigma} / \pi=\operatorname{Im}\left\{\int_{-a}^{a}\left[\sigma_{y y}^{\infty}(x, y) u_{y}^{\infty *}(x, y)+\sigma_{x y}^{\infty}(x, y) u_{x}^{\infty *}(x, y)\right] d x\right\}
$$

according to the anti-plane or in-plane problem to be considered. In such expressions, we define: $\tau_{\infty}=\mu\left(\partial w_{\infty} / \partial y\right), \sigma_{y y}^{\infty}=(\lambda+2 \mu)\left(\partial u_{y}^{\infty} / \partial y\right)+\lambda\left(\partial u_{x}^{\infty} / \partial x\right), \sigma_{x y}^{\infty}=$ $\mu\left[\left(\partial u_{x}^{\infty} / \partial y\right)+\left(\partial u_{y}^{\infty} / \partial x\right)\right][8]$, as well as $(\cdot)^{*}=$ complex conjugate of $(\cdot), \operatorname{Im}\{\cdot\}=$ imaginary part of $\{\cdot\}$.

By using (6.4), (6.5), (6.8), (6.9) and the above relations, equations (7.1a) and (7.1b) can be worked out in the two problems, to give

$$
E_{\infty}^{\tau} / \pi=\frac{1}{2 a \mu} \operatorname{Im}\left\{\frac{1}{q_{0}^{\tau}}\right\} \times\left(\begin{array}{cc}
\left|\Phi_{\varepsilon}\right|^{2} & , \varepsilon<1 / 2 \\
\left|\Phi_{+}\right|^{2}+\left|\Phi_{-}\right|^{2}, & \varepsilon=1 / 2
\end{array}\right)
$$

where for $\varepsilon \leq 1 / 2$ we have the following:

$$
\operatorname{Im}\left\{\frac{1}{q_{0}^{\tau}}\right\}=\left\{\begin{array}{c}
0, \quad a k / \pi<\varepsilon \quad\left(q_{0}^{\tau}>0: \text { no far-field propagation }\right) \\
1 / \sqrt{k^{2}-(\pi \varepsilon / a)^{2}}, \quad a k / \pi>\varepsilon \quad\left(q_{0}^{\tau}=-i \sqrt{k^{2}-(\pi \varepsilon / a)^{2}}: \text { far-field prop. }\right)
\end{array} ;\right.
$$

as well as

$$
\begin{gathered}
E_{\infty}^{\sigma} / \pi=\frac{k_{2}^{2}}{2 a \mu\left|\Delta_{0}\right|^{2}} \operatorname{Im}\left\{\left[k_{2}^{2}-2(\pi \varepsilon / a)^{2}\right]^{2}\left|e^{-q_{0}^{\sigma} y}\right|^{2} q_{0}^{\sigma *}+4(\pi \varepsilon / a)^{2}\left|e^{-r_{0}^{\sigma} y}\right|^{2}\left|q_{0}^{\sigma}\right|^{2} r_{0}^{\sigma *}\right\}_{y \rightarrow \infty} \\
\times\left(\begin{array}{c}
\left|\Psi_{\varepsilon}\right|^{2} \quad, \varepsilon<1 / 2 \\
\left|\Psi_{+}\right|^{2}+\left|\Psi_{-}\right|^{2}, \varepsilon=1 / 2
\end{array}\right),
\end{gathered}
$$


where for $\varepsilon \leq 1 / 2$ we have the following: $\operatorname{Im}\{\cdot\}_{y \rightarrow \infty}$

$$
=\left\{\begin{array}{l}
0, \quad\left(a k_{1} / \pi<\right) a k_{2} / \pi<\varepsilon \quad(I) \\
4(\pi \varepsilon / a)^{2}\left[(\pi \varepsilon / a)^{2}-k_{1}^{2}\right] \sqrt{k_{2}^{2}-(\pi \varepsilon / a)^{2}}, \quad a k_{1} / \pi<\varepsilon<a k_{2} / \pi \quad(I I) \\
{\left[k_{2}^{2}-2(\pi \varepsilon / a)^{2}\right]^{2} \sqrt{k_{1}^{2}-(\pi \varepsilon / a)^{2}}+4(\pi \varepsilon / a)^{2}\left[k_{1}^{2}-(\pi \varepsilon / a)^{2}\right] \sqrt{k_{2}^{2}-(\pi \varepsilon / a)^{2}}} \\
\varepsilon<a k_{1} / \pi<a k_{2} / \pi \quad(I I I)
\end{array}\right.
$$

where

(I) $\quad q_{0}^{\sigma}, r_{0}^{\sigma}>0$ : no far-field propagation,

(II) $q_{0}^{\sigma}>0, r_{0}^{\sigma}=-i \sqrt{k_{2}^{2}-(\pi \varepsilon / a)^{2}}$ : far-field prop. with only wave number $r_{0}^{\sigma}$,

(III) $q_{0}^{\sigma}=-i \sqrt{k_{1}^{2}-(\pi \varepsilon / a)^{2}}, r_{0}^{\sigma}=-i \sqrt{k_{2}^{2}-(\pi \varepsilon / a)^{2}}$ : far-field prop. with both wave numbers $q_{0}^{\sigma}, r_{0}^{\sigma}$.

For $\varepsilon>1 / 2$, we can use the expressions established for $\varepsilon<1 / 2$ by substituting in these $\varepsilon$ by $\varepsilon-1$ (so that $H_{0}, H_{\varepsilon}$ become $\hat{H}_{0}, \hat{H}_{\varepsilon-1}$ in (4.9) and (4.31)); of course, the inequalities involving $\varepsilon$ in $(7.2 \mathrm{~b})$ and $(7.3 \mathrm{~b})$ should be intended with $1-\varepsilon$ instead of $\varepsilon$.

We note that our mathematical procedure requires in the above formulas that $a k / \pi$ or $a k_{2} / \pi<1-\varepsilon$ when $\varepsilon<1 / 2, \quad a k / \pi$ or $a k_{2} / \pi<\varepsilon$ when $\varepsilon>1 / 2, \quad a k / \pi$ or $a k_{2} / \pi<3 / 2$ when $\varepsilon=1 / 2$; thus, the case of far-field propagation with both wave numbers (in the in-plane problem) can be included only if $k_{2} / k_{1}=\sqrt{(\lambda+2 \mu) / \mu}<$ $(1-\varepsilon) / \varepsilon$ when $\varepsilon<1 / 2, \quad k_{2} / k_{1}<\varepsilon /(1-\varepsilon)$ when $\varepsilon>1 / 2, \quad k_{2} / k_{1}<3$ when $\varepsilon=1 / 2$.

By choosing a fixed geometry $(b / a=1 / 2)$ and calculating all constants $H$ involved, we have derived the $\Phi$ 's and $\Psi$ 's by (4.9),(4.31) or (4.23),(4.41), and then we have plotted the energy at infinity versus the frequency parameter as given by the above explicit expressions; see Figs. 2-4. We took three values of the phase shift: $\varepsilon=1 / 4,3 / 4,1 / 2$.

First of all, we note that, as physically expected, the energy produced for $\varepsilon=3 / 4$ coincides with that for $\varepsilon=1 / 4$, since in this case $\hat{H}_{0}=H_{0}$ and $\hat{H}_{\varepsilon-1}=H_{\varepsilon}$ (see (6.1b),(6.2b $)_{1}$ and footnote 3 above); thus, we can unify the discussion for these two values.

In the anti-plane problem, after the beginning of the far-field propagation (with only the first mode), the behaviour of the energy is strictly increasing in the given range, contrary to the case of a null phase shift; moreover, the rate of increasing appears to be greater with some phase shift (in particular, with $\varepsilon=1 / 2$ ): compare lines 1 and 2 of Fig. 2 with line 3 of Fig. 2 in [7.

The situation is quite different in the in-plane problem, where the behaviour heavily depends on the physical properties of the material, more precisely on the value of the ratio $k_{2} / k_{1}=\sqrt{(\lambda+2 \mu) / \mu}$ with respect to the assumed phase shifts (see the remark above): when $k_{2} / k_{1}<3$, the far-field propagation begins (just after the critical value) with one wave number and a non-decreasing behaviour; then, at a frequency equal to $k_{2} / k_{1}$ times the critical value, the propagation with two wave numbers begins. See lines 1 in Figs. 3, 4. Approaching such a frequency, the energy shows a high peak immediately followed by a sharp dip to zero and another rapid increase (a new peak for $\varepsilon=1 / 2$ ); that is typical when resonance and/or anti-resonance effects occur at some frequency in the wave propagation. Such a behaviour is a specific characteristic of the phase shift case, 
since for $\varepsilon=0$ the far-field propagation (of the first mode) always occurs with only one wave number (the longitudinal one $k_{1}$ ) without oscillations [7. After this frequency, the energy meets a local minimum (very spread) to finally become slowly increasing. On the contrary, when $k_{2} / k_{1}>3$, the far-field propagation occurs with only one wave number throughout the given range; however, even if the energy does not show any oscillation, its behaviour is quite different from that of the case $\varepsilon=0$, which has a local maximum

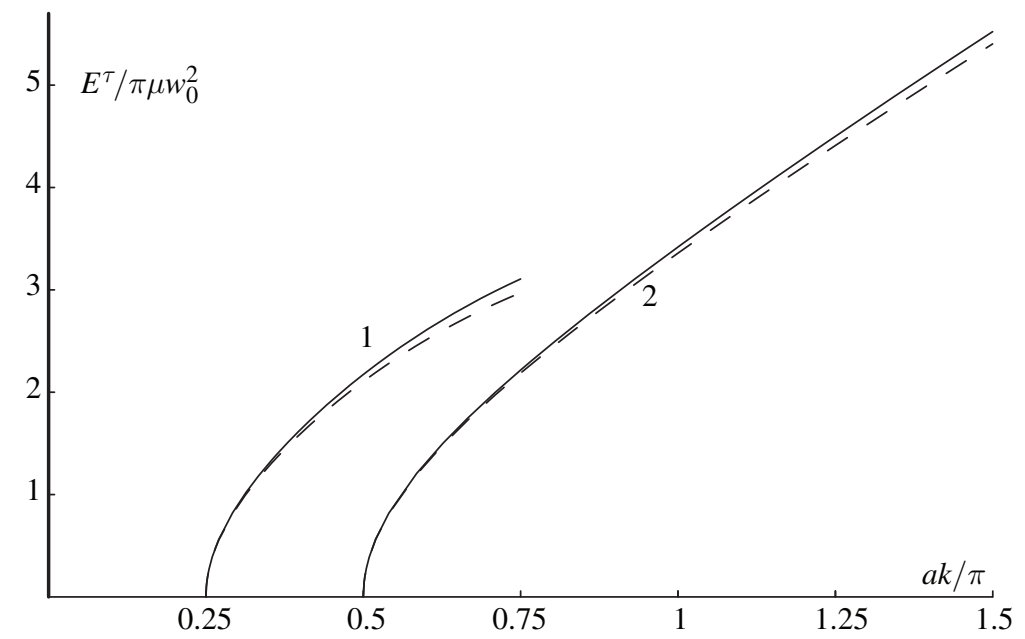

Fig. 2. For anti-plane problem, with $b / a=0.5$. Energy at infinity $E_{\infty}^{\tau} /\left(\pi \mu w_{0}^{2}\right)$ vs. $a k / \pi$. Line 1: $\varepsilon=1 / 4$ or $3 / 4, a k / \pi \in(0,3 / 4)$; line 2: $\varepsilon=1 / 2, a k / \pi \in(0,3 / 2)$. Dashed lines: exact numerical solution (at $y=0)$.

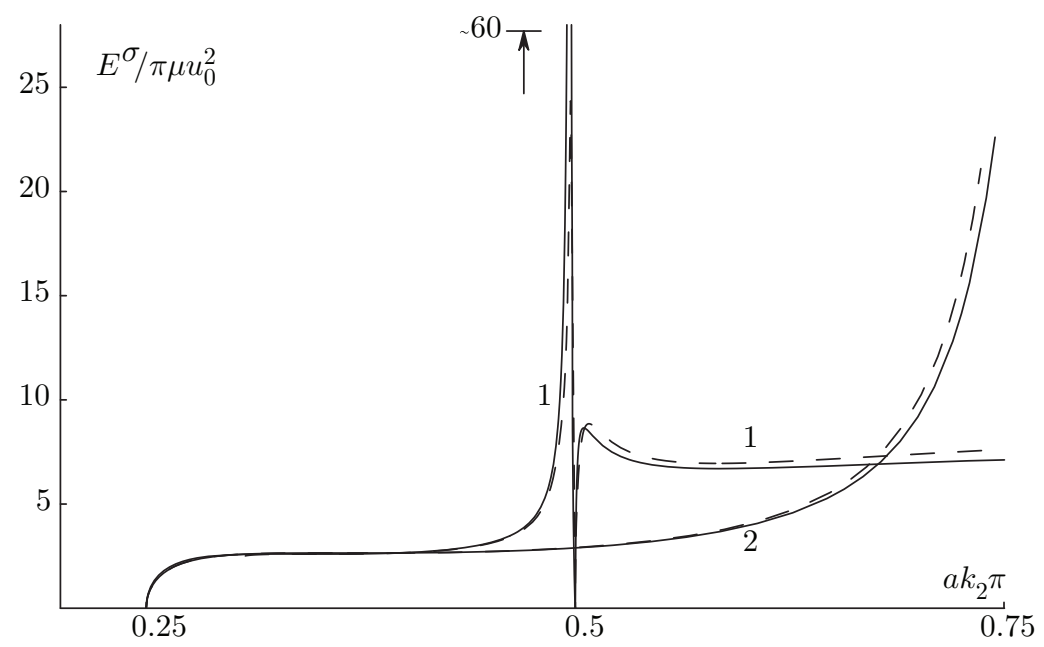

FIG. 3. For in-plane problem, with $b / a=0.5$ and $\varepsilon=1 / 4$ or $3 / 4$. Energy at infinity $E_{\infty}^{\sigma} /\left(\pi \mu u_{0}^{2}\right)$ vs. $a k_{2} / \pi \in(0,3 / 4)$. Line $1: k_{2} / k_{1}=$ 2; line 2: $k_{2} / k_{1}=4$. Dashed lines: exact numerical solution (at $y=0)$. 


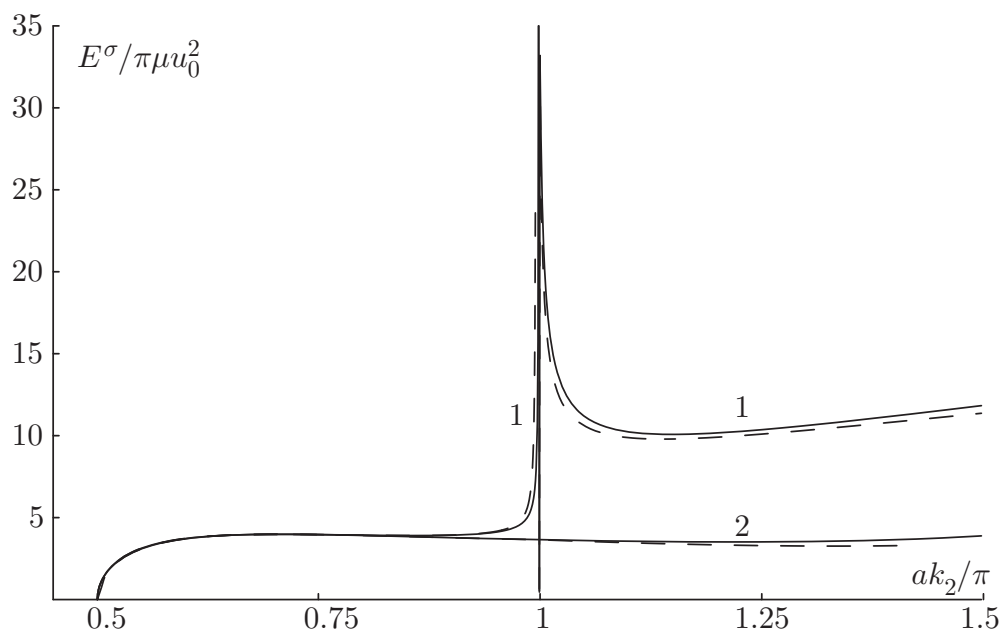

FIG. 4. For in-plane problem, with $b / a=0.5$ and $\varepsilon=1 / 2$. Energy at infinity $E_{\infty}^{\sigma} /\left(\pi \mu u_{0}^{2}\right)$ vs. $a k_{2} / \pi \in(0,3 / 2)$. Line $1: k_{2} / k_{1}=2$; line 2: $k_{2} / k_{1}=4$. Dashed lines: exact numerical solution (at $\left.y=0\right)$.

in the final part of the range: compare lines 2 of Figs. 3 and 4 with line 3 of Fig. 3 in [7.

On the surface of the medium (at $y=0$ ), the work over the geometric period $(-a, a)$ is simply given by

$$
\left(\begin{array}{l}
E_{0}^{\tau} / \pi \\
E_{0}^{\sigma} / \pi
\end{array}\right)=\operatorname{Im}\left\{\int_{-a}^{a}\left(\begin{array}{c}
\tau(x) w_{0}^{*}(x) \\
\sigma(x) u_{0}^{*}(x)
\end{array}\right) d x\right\}=\left(\begin{array}{l}
w_{0} \\
u_{0}
\end{array}\right) \operatorname{Im}\left\{\int_{-b}^{b}\left(\begin{array}{c}
\tau(x) \\
\sigma(x)
\end{array}\right) d x\right\},
$$

since the stresses are non-null only in the contact zone $(-b, b)$, and moreover (in the in-plane problem) only the normal component of the stress $\sigma=\sigma_{y y}$ is present in view of the friction-free boundary condition.

By solving numerically the integral equations (3.3),(3.6) with the exact kernels given in (3.5),(3.8), respectively, we have worked out the expressions (7.4) for the same values of geometric and physical parameters considered in the analytical procedure; the results are reported as dashed lines in the (corresponding) figures previously discussed. Of course, to plot now any curve with respect to frequency, those equations must be solved numerically each time anew for each new value of the frequency parameter. We can see that the agreement between exact numerical (at $y=0$ ) and approximate analytical (at $y \rightarrow \infty$ ) results is quite excellent, in particular for $\varepsilon=1 / 2$, apart from some (not large) discrepancies in the final parts of the frequency ranges involved. In our opinion, this fact can be used to confirm the validity of the procedure here developed.

\section{REFERENCES}

[1] K. Aki, P. G. Richards, Quantitative Seismology. Theory and Methods (1,2), Freeman: San Francisco, 1980. 
[2] J. Brouwer, K. Helbig, Shallow High-Resolution Reflection Seismics (Handbook of Geophysical Exploration: Seismic Exploration), Pergamon: New York, 1998.

[3] United States Patent 6028818 : Method and apparatus for multiple seismic vibratory surveys. (http://www.freepatentsonline.com/6028818.html)

[4] United States Patent 6807508 : Seismic prospecting method and device using simultaneous emission of seismic signals based on pseudo-random sequences. (http://www.freepatentsonline.com/6807508. html)

[5] United States Patent 6714867 : Method for seismic monitoring of an underground zone by simultaneous use of several vibroseismic sources. (http://www.freepatentsonline.com/6714867.html)

[6] J. J. Postel, E. Gillot, M. Larroque, Review of specific parameters in high-resolution seismic, EAGE 66th Conf., Paris, 2004, Z99. (http://www.freepatentsonline.com/6714867.html)

[7] E. Scarpetta, M. A. Sumbatyan, Wave Properties of the Elastic Half-space loaded by a Periodic Array of Vibrating Punches: an Analytical Approach, Adv. Theor. Appl. Mech.1 (6) 2008, 281-300.

[8] J. D. Achenbach, Wave Propagation in Elastic Solids, North-Holland: Amsterdam, 1973.

[9] A. P. Prudnikov, Y. A. Brychkov, O. I. Marichev. Integrals and Series (vol.2), Gordon and Breach Science Publishers: Amsterdam, 1986. MR.874987 (88f:00014)

[10] F. D. Gakhov, Boundary Value Problems, Pergamon Press: Oxford, 1966. MR0198152 (33:6311) 